\title{
Why does a mirror image look left-right reversed? A hypothesis of multiple processes
}

\author{
YOHTARO TAKANO \\ Waseda University, Tokyo, Japan
}

\begin{abstract}
This paper proposes a solution to the mirror reversal problem: Why does a mirror reverse left and right but not up and down? The paper first reviews past hypotheses and shows that none of them has succeeded in explaining all the related phenomena. It then proposes a multiprocess hypothesis based on the insight that what is called a mirror reversal is actually composed of three different types of reversal: The Type I reversal is produced by the discrepancy between an orientational framework that is aligned with a viewer's body and the one that is assumed in the viewer's mirror image; the Type II reversal is produced by the discrepancy between the mental representation of an object and its mirror image; and the Type III reversal is produced by a mirror's optical transformation. The proposed hypothesis is shown to provide reasonable accounts for all the related phenomena disputed in the past literature.
\end{abstract}

When a viewer faces a plane mirror, alphanumeric characters are illegible because their left and right sides are reversed; a watch around the viewer's left wrist is seen on the right wrist of the viewer's own mirror image. Why does a mirror reverse left and right but not up and down? Although a number of psychologists, philosophers, physicists, and mathematicians have been discussing this mirror reversal problem for nearly half a century, no satisfactory answer has ever been obtained (Ittelson, Mowafy, \& Magid, 1991; Morris, 1993).

This paper first reviews major hypotheses that have been presented to answer this problem, along with criticisms raised against them. It then proposes a multiprocess hypothesis and shows how it explains the mirror reversal and related phenomena. It will also be shown that the proposed hypothesis is invulnerable to those criticisms.

\section{PAST HYPOTHESES}

In the past literature, six relatively independent principles of explanation can be identified, though some of them may be combined to provide a single answer. This section will examine how each principle was employed to form an answer to the mirror reversal problem and how it failed.

Before reviewing the preceding hypotheses, it seems appropriate to examine the view that there is no real problem to be solved. This view asserts that the mirror reversal problem is a pseudoproblem because a form and its mirror image are geometrically isomorphic in that they can be brought into coincidence by appropriate geometric operations. According to this line of argument, however, one

Y.T. is grateful to Ulric Neisser for his warm support concerning the present paper. Correspondence should be addressed to Y. Takano, Department of Psychology, Graduate School of Humanities and Sociology, University of Tokyo, Bunkyo-ku, Tokyo 113-0033, Japan (e-mail: ltaro@hongo.ecc.u-tokyo.ac.jp). could maintain that the Müller-Lyer illusion, for example, is not worth investigating because the two compared lines are identical in length from a geometrical point of view. The mirror reversal problem arises from a discrepancy in recognized directions, just as the Müller-Lyer illusion arises from a discrepancy in recognized length. From a psychological point of view, it is undeniable that the former discrepancy as well as the latter exists in reality. Therefore, the mirror reversal problem is not a pseudoproblem, but a real psychological problem awaiting an explanation.

\section{Optical Hypothesis}

Recently, Haig (1993) maintained that basic optics is sufficient to solve the mirror reversal problem because it is the optical characteristic of a mirror to reverse left and right without reversing up and down. In Figure 1, the eye of a viewer is located at $\mathrm{E}$. Haig claimed that looking from $\mathrm{E}$ is equivalent to looking from $\mathrm{F}$, which is a hypothetical viewpoint assumed behind the mirror. When the eye looks from $F$ at the mirror image $(P Q)$ of the vertical line segment $(\mathrm{AC})$, the top and bottom are not reversed (Figure 1A). By contrast, when the eye looks from $\mathrm{F}$ at the mirror image (ST) of the horizontal line segment (BD), the left and right are reversed (Figure 1B). According to Haig, the mirror thus optically reverses left and right without reversing up and down.

Morris (1993) pointed out that left and right are not reversed at $\mathrm{E}$, where the eye is actually located. That is, $F$ is not equivalent to $E$ in this critical respect. In addition, the left-right reversal takes place at $\mathrm{F}$ only when the viewpoint moves from $\mathrm{E}$ to $\mathrm{F}$ by a $180^{\circ}$ rotation about a vertical axis. If the viewpoint moves by a $180^{\circ}$ rotation about a horizontal axis, the left-right reversal does not take place. Instead, an up-down reversal emerges. Optics does not explain why the viewpoint has to move from $\mathrm{E}$ to $\mathrm{F}$ and why that move has to be performed about a vertical axis instead of a horizontal axis. 

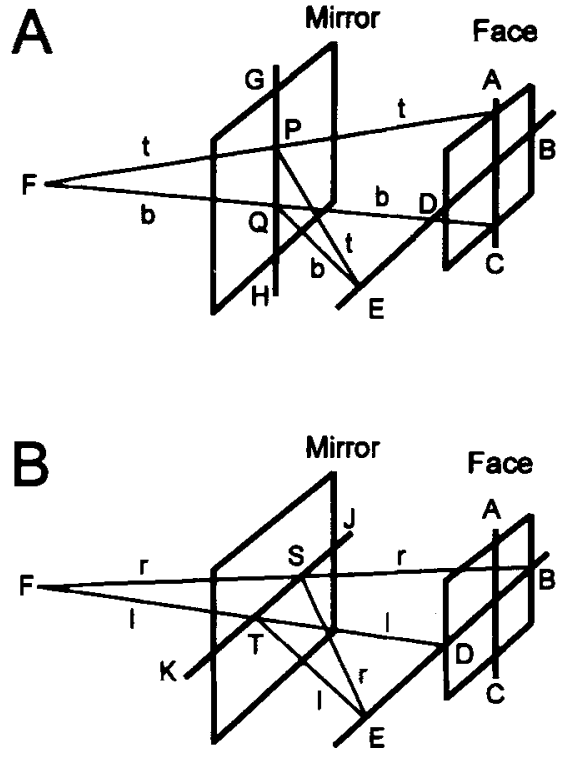

Figure 1. Optical hypothesis. The "face," ABCD, is reflected by the mirror; the viewer's eye is located at $\mathrm{E}$. (A) The mirror image, $P Q$, of the vertical line segment, $A C$, is not inverted when seen from the hypothetical viewpoint, F, which Haig (1993) supposed to be equivalent to $E$. (B) The mirror image, ST, of the horizontal line segment, $\mathrm{BD}$, is reversed with respect to left and right when seen from F. Note-From "Reflections on Inversion and Reversion," by N. D. Haig, 1993, Perception, 22, p. 864. Copyright 1993 by Pion Ltd. Adapted with permission.

Another criticism can be raised against Haig's (1993) explanation. If his explanation were correct, an up-down reversal would be observed by the eye located on the elongation of $\mathrm{AC}$ in Figure $1 \mathrm{~A}$ because the optical layout becomes essentially identical to that in Figure 1B, producing what Haig called a "sagittal view" (pp. 864865 ). In actuality, however, the predicted up-down reversal is not recognized. The reader can easily confirm this by looking at the mirror image of a pencil that is held vertically in front of a mirror while locating the eye above it. What is more, even if the pencil is aligned with a horizontal line (BD in Figure $1 \mathrm{~B}$ ) so that it points to the right, its mirror image is still pointing to the right and no left-right reversal is observed.

In conclusion, optics alone is not sufficient to understand why left and right are reversed selectively by a mirror. This failure of the optical hypothesis endorses the view that the explanation of the mirror reversal has to take psychological processes into consideration.

As for the optical property of a mirror, all researchers except Haig (1993) agree that it reverses neither left and right nor up and down (e.g., Block, 1974; Gardner, 1990; Morris, 1993; Shepard \& Hurwitz, 1984). They also agree that it is only front and back that are actually reversed by the optical property of a plane mirror. Suppose that objects $\mathrm{A}$ and $\mathrm{B}$ are located between a mirror and a viewer and that $\mathrm{A}$ is closer to the viewer than is $\mathrm{B}$. In their mirror image, the viewer sees the converse: That is,
B is closer than A. This is because a plane mirror reverses an optical layout along the axis that is perpendicular to its surface. When a viewer faces a mirror, therefore, only the front-back axis is reversed. In the following discussion, what is presupposed about the optical property of a plane mirror will be confined only to this agreedupon fact.

Incidentally, a mirror does not actually reverse a frontback axis because it is virtually two dimensional and thus does not have a front-back dimension. The "reversal of a front-back axis" simply means that a plane mirror transforms an optical layout so that the perceived depth in its mirror image is reversed.

\section{Locomotion Hypothesis}

Pears (1952) assumed that a viewer always imagines "getting into" his/her own mirror image. The ordinary way of locomotion for humans is walking on a horizontal surface. Accordingly, the viewer imagines a rotational locomotion about a vertical axis to "get into" the mirror image. As a result, the head and feet of the imagined viewer's body are superimposed onto the head and feet of the mirror image, and the face and back of the imagined body are superimposed onto the face and back of the mirror image. However, the left hand of the imagined body is superimposed onto the mirror image's hand, which is on the right from the real viewer's viewpoint; the right hand of the imagined body is superimposed onto the mirror image's hand, which is on the left from the real viewer's viewpoint. Thus, a left-right reversal is recognized.

Block (1974, p. 263) pointed out that Pears (1952) failed to explain why the locomotion has to be the rotation. If the viewer imagines walking straight ahead to get into the mirror image, the front and back are reversed instead of the left and right. Walking straight ahead is an ordinary type of locomotion for humans.

Navon (1987, p. 280) presented a different criticism. Pears's (1952) hypothesis predicts that when a viewer lies down on his/her side while facing a mirror, he/she should recognize an up-down (i.e., head-feet) reversal because the rotation about a vertical axis causes the head of the imagined body to be superimposed onto the feet of the mirror image. In actuality, however, the left-right reversal is still recognized, although the left-right axis is aligned with the direction of gravity in this case. (Mayo, 1958, p. 114 , made essentially the same point.)

Navon (1987, p. 280) presented still another criticism. When a viewer looks down at a mirror on a floor or looks up at a mirror on a ceiling, a left-right reversal is recognized. If the viewer imagines the rotational movement of the body about a vertical axis, however, the imagined body cannot be superimposed onto the mirror image in such a way that the right hand of the imagined body coincides with the mirror image's hand, which is on the left from the real viewer's viewpoint.

Finally, Pears (1952) did not answer the question of why a viewer has to imagine superimposing his/her own body onto its mirror image. Recent studies of form recog- 
nition have confirmed that superimposing an image is not necessary for recognizing the form of an object (e.g., Biederman, 1987; Marr, 1982; Takano, 1989). This problem is especially conspicuous when a viewer sees the mirror image of an alphanumeric character. Why does the viewer have to imagine to superimpose his/her own body onto the character?

\section{Symmetry Hypothesis}

Pears (1952) presented another reason for the rotation of the viewer's body image: It is rotated about a vertical axis because the discrepancy between the body image and the mirror image as a result of the superimposition is minimized. It is the approximate bilateral symmetry of a human body that causes the rotation about a vertical axis to produce the minimal discrepancy.

This symmetry hypothesis is supported by the majority of researchers (e.g., Corballis \& Beale, 1976; Ittelson et al., 1991; Mayo, 1958; Shepard \& Hurwitz, 1984). It circumvents three of the criticisms raised against the locomotion hypothesis. First, it provides a convincing reason for the rotational movement: Rotational movement produces minimal discrepancy, whereas the superimposition by moving straight ahead produces a much larger discrepancy. Second, this hypothesis explains why a left-right reversal is recognized when a viewer is lying down in front of a mirror: In this case, a rotation about a horizontal axis, which results in a left-right reversal, produces minimal discrepancy, whereas a rotation about a vertical axis produces a larger discrepancy. Finally, this hypothesis explains in a similar fashion why a left-right reversal is recognized when a viewer looks down at a mirror on a floor or looks up at a mirror on a ceiling: In these cases, a rotation about a front-back axis on the surface of the mirror, which results in a left-right reversal, produces a minimal discrepancy, whereas a rotation about a vertical axis produces a larger discrepancy.

However, the symmetry hypothesis has to face a new criticism as well as the rest of the earlier ones. First, Navon $(1987$, p. 281$)$ contended that "a congenitally asymmetric cripple" would recognize a left-right reversal, whereas the symmetry hypothesis predicts that a person with this kind of handicap would not recognize a left-right reversal because the discrepancy is not minimal after a rotation about a vertical axis. Second, this hypothesis does not answer the question of why an image has to be superimposed at all. Related to this question, the symmetry hypothesis shares with the locomotion hypothesis the weakness that the left-right reversal of an alphanumeric character is not readily comprehensible. There is no reasonable basis for assuming that a viewer routinely superimposes the image of his/her own body onto a character or a string of characters.

One might argue that this hypothesis could be modified to accommodate the case of a character: When a viewer recognizes the mirror image of a character, what the viewer rotates is not the viewer's body image but the image of the original character, which faces the mirror image. However, this modification leads to a fatal contradiction. Suppose, for example, that a viewer is looking at the mirror image of a character, $\mathrm{C}$, which is approximately symmetric with respect to a horizontal axis but obviously asymmetric with respect to a vertical axis. The modified symmetry hypothesis predicts that an updown reversal should be recognized because the rotation of the original character's image about a horizontal axis produces a minimal discrepancy when it is superimposed onto its mirror image. In actuality, however, this is a typical case in which a left-right reversal is recognized.

\section{Conventional Description Hypothesis}

Gardner (1964) wrote, "Such a reversal [mirror reversal] automatically changes an asymmetric figure to its enantiomorph. Because we ourselves are bilaterally symmetrical, we find it convenient to call this a leftright reversal. It is just a manner of speaking, a convention in the use of words" (p. 31 ; enantiomorph means a left-right reversed shape; see the section on the Type IIIII reversal for a more precise definition). As Gardner (1990) has pointed out, this conventional description hypothesis has gained support from several researchers (e.g., Bennett, 1970; Corballis \& Beale, 1976).

However, this hypothesis is not explicit as to the reason why the approximate bilateral symmetry of a human body causes the alleged verbal convention - a left-right reversal. The cited researchers have pointed out that the left-right direction of a mirror image can be determined only after the up-down and front-back directions are determined because the left-right direction is ambiguous due to the approximate bilateral symmetry. Why is the left-right direction ambiguous and why are the up-down and front-back directions unambiguous? Is it because a viewer assigns the directions to the mirror image so that the discrepancy between the mirror image and the prototypical form of a human body becomes minimal? If so, this hypothesis is virtually identical to the symmetry hypothesis. According to the symmetry hypothesis, however, the left-right reversal is supposed to be the inevitable consequence of form recognition process and not "just a manner of speaking." Therefore, the conventional description hypothesis is not identical to the symmetry hypothesis. However, the cited researchers have not specified in other ways how the alleged verbal convention is related to the approximate bilateral symmetry of a human body. It is also unclear why the verbal convention has to be a reversal of left and right in the case of a mirror image but a nonreversal in the case of a real body, which is also approximately bilaterally symmetrical. Until these questions are answered, it is impossible to judge whether or not the conventional description hypothesis possesses explanatory power.

This hypothesis has been questioned in other respects as well. Block (1974, pp. 274-276) criticized Gardner (1964) and Bennett (1970) by highlighting the case of a side view in which a viewer "stands shoulder to a mirror" so that a line assumed to pass through the two shoulders 
becomes perpendicular to the mirror's surface. Both Gardner and Bennett claimed that a true left-right reversal takes place in this case. Block pointed out that they failed to explain why the left-right reversal is conventional and true in the case of the side view and conventional but false in the case of the frontal view.

Navon (1987) highlighted the cases of a curved mirror, two plane mirrors, and three plane mirrors. The conventional description hypothesis predicts that the recognized reversal should always be left-right because the verbal convention has been established as "a left-right reversal." In those mirrors, however, a viewer recognizes no reversal, an up-down reversal, or both left-right and up-down reversals (p. 279). This strongly suggests that the true basis of a recognized left-right reversal is not the mere verbal convention but rather the nature of a reversal that is actually produced in the process of recognizing a mirror image.

In a specific setting, an up-down reversal alone can be observed without a left-right reversal. Put a mirror on a table and hold a sheet of paper above it so that the paper is roughly perpendicular to the mirror. Then, an alphanumeric character on the paper will look upside down in the mirror, but its left and right sides are not reversed. This inversion occurs when we see ponds and lakes: Mountains, trees, birds, and people all look upside down. This phenomenon questions the claim that something specific to left and right (e.g., the approximate bilateral symmetry of a human body) is responsible for the mirror reversal. This case and those presented by Navon (1987) can be taken as evidence against the symmetry hypothesis as well because it, too, is based on the approximate bilateral symmetry of a human body.

Meanwhile, Gardner (1990) contended that it is the terms left and right that cause the false conventional description "left-right reversal." He proposed, "This confusion vanishes in the more precise language of 3-space coordinate geometry, where there is no distinction between the coordinates except that they are called $x, y$, and $z$ " (p. 22). It is questionable that this proposal would dissolve the mirror reversal problem. The coordinates need graduations as in the case of an ordinary Cartesian coordinate system, in which each axis has an origin, a plus area, and a minus area, along with increasing absolute values in both directions; otherwise, two directions could not be discriminated along the axis. Suppose that left and right were replaced by $x$. Then, a left-right reversal would be simply replaced by a plus-minus reversal in the value of $x$. No essential difference would be made. Thus, the proposed modification in terminology does not resolve the mirror reversal problem.

\section{Frontal Encounter Schema Hypothesis}

Navon (1987) assumed that a viewer forms a frontal encounter schema on the basis of frequent typical encounters in which the intrinsically defined fronts of two objects face each other. This schema gives rise to the expectation that the viewer's head should face the other's head and the viewer's feet should face the other's feet. It also gives rise to the expectation that the viewer's right hand should face the other's left hand and the viewer's left hand should face the other's right hand. When the viewer faces a mirror to see the mirror image of his/her own body, the situation resembles a typical frontal encounter. Consequently, the viewer invokes the frontal encounter schema, which leads to the expectations above. Although the former expectation is satisfied, the latter is not "because that schema leads you to expect the right hand of the image to face your left hand, but you see that actually what faces your left hand, hence should have been the image's right hand, is rather the reflection of your left hand" (p. 277). Thus, a left-right reversal is recognized.

This explanation has at least two difficulties. First, when a viewer looks at a mirror on a floor or a ceiling, the frontal encounter schema will not be invoked because this setting, in which two bodies are inverted in relation to each other, is distinct from the typical frontal encounter. Therefore, the frontal encounter schema hypothesis predicts that a left-right reversal will not be recognized. In actuality, however, it is recognized, as Navon (1987, p. 280) himself pointed out.

Second, this hypothesis has difficulty in accounting for the left-right reversal of an alphanumeric character. The left and right sides of a character are not intrinsically defined in contrast to those of a human body. In many cases, they are defined on the basis of a reader's viewpoint. An uppercase letter, $C$, for instance, is usually said to have an opening on the right, not on the left. When a viewer reads a character, therefore, its left faces the viewer's left and its right faces the viewer's right. The frontal encounter schema does not apply because it assumes the converse: That is, the character's left should face the viewer's right and the character's right should face the viewer's left. Therefore, the frontal encounter schema hypothesis does not predict a left-right reversal for the mirror image of a character. In actuality, the leftright reversal of a character is evident.

\section{Rotation Hypothesis}

Gregory (1987, p. 492) attempted to explain the mirror reversal problem in terms of the physical rotation of an object. To see an object in a mirror, the object has to be rotated from a viewer's direct view to face the mirror. This rotation is ordinarily conducted on a vertical axis. As a consequence, the object's left and right sides in the direct view are reversed in its mirror image. When a viewer sees a room in a mirror, it is not the room but rather the viewer that is rotated.

This hypothesis has at least four difficulties. First, it does not explain the left-right reversal of a viewer's own body. In the rotation hypothesis, a left-right reversal is supposed to arise from a comparison between the direct view of an object and its mirror image. However, the direct view from the outside of the viewer's body is not available for the viewer. Hence, no comparison can be made. Second, no object is physically rotated in many 
cases. When a left-right reversal is recognized in the mirror image of a viewer's own body, no rotation of the viewer's body is involved. Furthermore, the mirror reversal of an alphanumeric character can be recognized without rotating anything in some cases. Suppose that a viewer in the driver's seat of a car sees characters of a billboard in a rearview mirror without seeing their original beforehand. The left-right reversal in the mirror will be compelling nevertheless. In this case as well, no physical entity is rotated. Third, the physical rotation of an object actually shows its rear side to a viewer rather than showing its enantiomorph. When a book is rotated, for example, its back cover is seen instead of the enantiomorph of its front cover. Finally, the physical rotation of an object causes a front-back reversal: The rear side becomes closer to a viewer than the front side, whereas the front side was closer before the rotation. By contrast, no front-back reversal is observed in the mirror image: The front side remains closer to the viewer, as in the direct view. The last two problems suggest that physical rotation alone does not provide a satisfactory explanation for the mirror reversal even in those cases where the rotation hypothesis appears to hold.

\section{MULTIPROCESS HYPOTHESIS}

Although the preceding hypotheses explain certain aspects of the mirror reversal, none of them has succeeded in explaining all the related phenomena within a consistent theoretical framework. This section will present a multiprocess hypothesis to provide consistent accounts for all these phenomena. This hypothesis is based on the critical insight that what is called a mirror reversal is actually composed of three different types of reversal. The Type I reversal is primarily related to a viewer's own body and emerges psychologically from the comparison between the real body and its mirror image. The Type II reversal is primarily related to alphanumeric characters and emerges psychologically from the comparison between their mental representations and mirror images. These two types of mirror reversal are also different from each other with respect to orientational frameworks used to judge the directions of the mirror images. The Type III reversal is related to both a viewer's body and characters and emerges optically, not psychologically, from the comparison between a real object and its mirror image.

By attending to the following fact one will intuitively realize that Type I and Type II reversals take place for different reasons. Suppose that a viewer with a watch around his/her left wrist faces a mirror. The viewer's mirror image has a watch around the right wrist-hence a leftright mirror reversal. However, this "right" is the direction that is judged from the mirror image's viewpoint. By contrast, the real viewer has the watch on the left, which is judged from the real viewer's viewpoint. If the direction of the mirror image is likewise judged from the real viewer's viewpoint, the mirror image watch is also on the left-hence no reversal in direction. In short, the leftright mirror reversal is recognized only when the left and right of the mirror image are judged from the mirror image's viewpoint, whereas those of the real viewer are judged from the real viewer's viewpoint. In the case of characters, however, the left-right mirror reversal is recognized when the left and right of a real character and those of its mirror image are both judged from the real viewer's viewpoint. We do not have to assume the mirror image character's viewpoint. Thus, it is evident that the mirror reversal of a viewer and that of a character are produced by different underlying principles.

\section{Orientational Frameworks}

To identify those underlying principles, it is necessary to clarify exactly what "viewpoint" means. In the preceding discussion, "viewpoint" is synonymous with an orientational framework because it is used only to judge directions. Accordingly, the orientational frameworks have to be specified first.

Generally speaking, the specification of orientational frameworks is critical whenever mirror images are discussed. A form and its mirror image are different from each other only in orientational relationships among the figural elements that constitute each form (Takano, 1989). Imagine, for example, an uppercase letter, $L$, and its mirror image. They are different from each other only in that the shorter line is to the right of the longer one in the former, whereas the shorter line is to the left of the longer one in the latter. Nothing else is different. However, these orientational relationships cannot be determined until a certain orientational framework is given. Neither left nor right is defined without it. This is why the specification of orientational frameworks is indispensable.

To understand the nature of the difference between Type I and Type II reversals precisely, it is necessary to distinguish between two types of orientational framework--one for perceptual encoding (the perceptual frame) and the other for cognitive representation (the representational frame). As shown below, this distinction is consistent with what is commonly known about form recognition and cognitive maps.

In an effort to explicate the process of form recognition, Marr (1982; Marr \& Nishihara, 1978) proposed that the human visual system employs two types of orientational framework - a viewer-centered coordinate system and an object-centered coordinate system. When a viewer is perceiving an object, its visual information is encoded in reference to the viewer-centered coordinate system (referred to here as the "perceptual frame"). The perceptual frame is always aligned with the viewer's body: The "up" of the perceptual frame corresponds to the direction of the head, its "down" to that of the feet, and so on. The perceptual frame moves together with the viewer's body. Accordingly, the description of an object's form in reference to the perceptual frame undergoes substantial modification when the viewer's position changes in relation to the object. 
Marr (1982; Marr \& Nishihara, 1978) introduced another orientational framework-the object-centered coordinate system - to explain the fact that the human visual system is able to recognize the form of an object irrespective of the relative orientational change between the viewer and the object as well as the resultant change in the description of its form in reference to the perceptual frame. The object-centered coordinate system is assumed inside each object and independent of the viewer's body. Therefore, the description of the object's form in reference to the object-centered coordinate system remains identical regardless of the relative orientational change between the viewer and the object. Thus, it is supposed to serve as a basis of stable form recognition.

In some cases, however, human form recognition cannot avoid the influence of an orientational change (see, e.g., Rock, 1973; Rock, Schreiber, \& Ro, 1994; Shepard \& Metzler, 1971). The object-centered coordinate system does not provide a reasonable account for such an influence. Takano (1989) showed that both presence and absence of the influence of an orientational change can be explained by substituting a subject-centered coordinate system (referred to here as the "representational frame") for the object-centered coordinate system. This subject refers to a subject who constructs the cognitive representation of an object's form. While the perceptual frame is used for perceptual encoding of an object, the representational frame is used for constructing its cognitive representation, which can be retrieved and processed later. Both frames coincide with each other when a viewer is actually perceiving an object and constructing its cognitive representation on the basis of its perceptual input. At this time, therefore, the directions defined by the representational frame are identical to those defined by the perceptual frame. Both frames are bound to the specific viewpoint that the viewer's body actually takes while perceiving.

Although the representational frame can be used to construct a cognitive representation on the basis of perceptual input, it can also be used to locate the retrieved representation of an object in representational space. Suppose, as an illustration, that a viewer is looking at a door that has a knob on the right. At this time, the viewer perceives the door on the basis of the perceptual frame and constructs its cognitive representation on the basis of the representational frame. Suppose that the viewer has turned around. Both the perceptual and representational frames have been rotated along with the viewer's body. Now the viewer cannot perceive the door. Therefore, the current perceptual frame cannot be applied to it. Nevertheless, the viewer could represent the door on the basis of the current representational frame. In other words, the viewer could imagine the door behind at the very place where the real door is located. Incidentally, a cognitive map thus constructed in representational space makes it easier for humans and animals to move around in and act on their environments (see, e.g., Gallistel, 1994; Golledge, 1986; Kozlowski \& Bryant, 1977). It is worth noting that the represented door has its knob on the left when judged by the current representational frame, whereas the knob was seen on the right when the door was actually perceived. This left-right reversal was produced by the turn of the perceptual and representational frames, concomitant with that of the viewer.

The retrieved representation can be located at any other place. For instance, the viewer who has turned around could retrieve the appearance of the door in front. In this case, what is recognized on the basis of the current representational frame (i.e., either an abstract representation or a concrete mental image of the door) is entirely different from what is being perceived on the basis of the current perceptual frame. In addition, the retrieved knob is on the right, not on the left, when judged by the current representational frame. This position of the knob in relation to the door is identical to that of the actually perceived knob. Incidentally, this preservation of a direction plays a critical role in the Type II mirror reversal.

Thus, the perceptual and representational frames could perform very different functions while both of them are aligned with the viewer's body. Furthermore, the representational frame can be rotated away from the perceptual frame. Hinton and Parsons (1981), for example, demonstrated that an orientational framework, instead of a mental image, could be rotated to discriminate misoriented mirror images from their originals under certain optimal conditions.

The representational frame can even be separated from the viewer's body entirely. Take the three-mountains task (Piaget \& Inhelder, 1948/1956) as an instance. In this task, a viewer looks from an indicated viewpoint at a miniature containing three mountains, which have different sizes, colors, and attachments. The viewer is asked to construct another miniature to reproduce the original while assuming that it is seen from a different indicated viewpoint. Preschoolers tend to reproduce the miniature erroneously from the actual viewpoint that they are taking to see the original, whereas adults usually succeed in reproducing the miniature from the indicated different hypothetical viewpoint. This suggests that adults are able to transform the current representational frame to recognize from a hypothetical viewpoint the representation that is constructed on the basis of the current perceptual input. After this transformation has been conducted, the current representational frame no longer coincides with the current perceptual frame, which is always bound to the viewer's body. Incidentally, this transformation of the representational frame plays a critical role in the Type I mirror reversal.

\section{Type I Reversal}

This section will show how the multiprocess hypothesis explains the Type I reversal. Suppose that a viewer with a watch around his/her left wrist is looking at the mirror image of his/her own body. A mirror reversal is recognized between the real body and its mirror image. This means that the viewer compares the directions of 
his/her real body with those of the mirror image. As pointed out earlier, the directions of the mirror image are judged from the mirror image's viewpoint, not from the viewer's. To take the viewpoint of the mirror image, the viewer has to rotate the representational frame alone about a vertical axis through $180^{\circ}$, leaving the perceptual frame in the real body (Figure 2). This rotation will be referred to as "representational transformation" because it is conducted in the representational space. No physical entity is rotated. The viewer judges that the watch of the mirror image is on the right in reference to the rotated representational frame. At the same time, the viewer judges that the real watch is on the left in reference to the perceptual frame, which is aligned with the real body. The viewer will then recognize that left and right are reversed between these two judgments. The Type I reversal is thus explained.

The position of the real watch can be recognized through tactual sensation or memory. Generally, the position of an object attached to the viewer's own body can also be recognized through visual perception of the object's mirror image combined with reasoning that utilizes the general knowledge about mirror reflection (e.g., "The real watch must be on the left because its mirror image is seen on the left, and the mirror image's left is the reflection of the real object's left if the directions are consistently judged from the real body's viewpoint."). The position of a body part can be often recognized through proprioception as well (e.g., when a viewer winks).

To explain the Type I reversal precisely, the optical transformation by the mirror has to be taken into account. As stated earlier, the mirror reverses an optical layout along an axis perpendicular to its surface. This

\section{Representational}

Frame

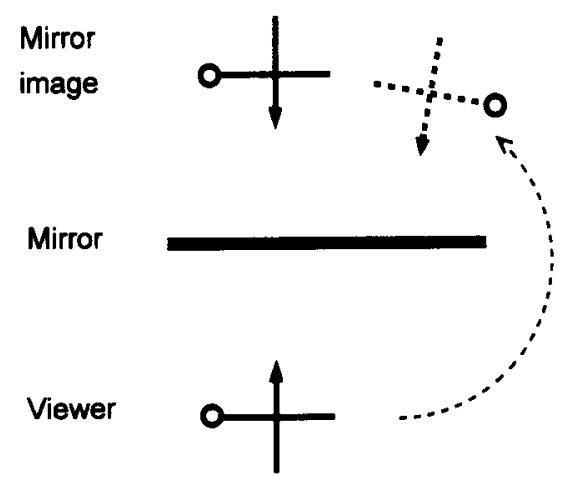

Figure 2. Type I reversal. The scene is sketched as it is viewed from above. The viewer as well as his/her mirror image is indicated by a cross drawn with solid lines. The viewer faces in the direction indicated by the arrow and is supposed to have a watch around the left wrist, which is indicated by the circle. To judge directions from the mirror image's viewpoint, the viewer rotates the representational frame alone, which is indicated by the cross drawn with dotted lines, so that it is aligned with the mirror image. The viewer then recognizes that the mirror image has the watch around the right wrist, whereas the real viewer has the watch around the left wrist.
Table 1 Type I Reversal

\begin{tabular}{|c|c|c|c|}
\hline \multirow[b]{2}{*}{$\begin{array}{l}\text { Form } \\
\text { tional }\end{array}$} & \multicolumn{3}{|c|}{ Frame } \\
\hline & Perceptual & & Representa \\
\hline Real object & $\mathrm{U}-\mathrm{D} / \mathrm{F} \downarrow \mathrm{B} / \mathrm{L}-\mathrm{R}$ & & \\
\hline Mirror image & $\mathrm{U}-\mathrm{D} / B-F / \mathrm{L}-\mathrm{R}$ & $\rightarrow$ & $\mathrm{U}-\mathrm{D} / \mathrm{F}-\mathrm{B} / R-L$ \\
\hline Comparison & & & $(-) /(-) /(\mathrm{R})$ \\
\hline
\end{tabular}

Note-The uppercase letters indicate the directions first ascribed to the real object by the perceptual frame: U-D, up-down; F-B, front-back; $L-R$, left-right. The reversal of each direction is indicated by reversing an order (e.g., $\mathrm{L}-\mathrm{R}$ vs. $R-L)$. (R), reversal; $(-)$, nonreversal in the final comparison.

optical transformation can be explained by optics alone. When the viewer faces the mirror, the front-back direction is reversed because it is perpendicular to the mirror.

Table 1 shows how the combination of the representational and optical transformations produces the left-right mirror reversal. In this table, the hyphenated uppercase letters (e.g., L-R) in each cell indicate the directions (e.g., left-right) initially ascribed to the form of a real object. The order of the letters indicates the relationship between those initial directions and the axes of the current frame that is used to judge directions at a given moment. The current frame is indicated under the column spanner Frame in Table 1 (i.e., the perceptual or representational frame). The italicized reversed order (e.g., $R-L$ ) indicates that the relationship is reversed (e.g., the initial left of the form is now on the right if judged by the current frame).

Initially, the directions are ascribed to the real body by the perceptual frame (see the Real object $\times$ Perceptual cell in Table 1). When it is reflected in the mirror, this optical transformation reverses only the front-back direction of the mirror image, as usual, when the directions are judged by the perceptual frame (see the Mirror image $\times$ Perceptual cell). In other words, after identifying the body parts of the mirror image through a form recognition process, the viewer recognizes that the mirror image is looking toward the "back" for the real viewer. The other directions remain unchanged: In the mirror image, the head is still above the feet, and the hand with the watch is still on the left if judged from the real viewer's viewpoint or the perceptual frame.

To assume the mirror image's viewpoint, the viewer rotates the representational frame alone about a vertical axis through $180^{\circ}$, leaving the perceptual frame in the real body. This representational transformation reverses both front-back and left-right axes of the representational frame if compared with those of the perceptual frame; the up-down axis remains unchanged. These consequences can be explained in terms of elementary geometry. When the directions of the mirror image are judged by this rotated representational frame, however, its already reversed front-back direction is no longer reversed because it is judged by the reversed front-back axis. Only the left-right direction is judged to be reversed because it has not been reversed by the optical transforma- 
tion (see the Mirror image $\times$ Representational cell). Thus, the watch of the mirror image is judged to be on the right by the rotated representational frame. When the viewer compares the directions of the mirror image judged by the rotated representational frame with those of the real body judged by the perceptual frame, therefore, the leftright direction alone is reversed (see the Comparison $x$ Representational cell). The Type I reversal is thus explained while taking the optical transformation into account. Ironically, the so-called left-right mirror reversal occurs precisely because the mirror does not reverse left and right.

The multiprocess hypothesis resembles the locomotion hypothesis and the symmetry hypothesis in assuming the rotational transformation of the representational frame. Although, in most preceding articles, the two hypotheses do not refer to it explicitly and simply refer to the rotation of the viewer's body image, the rotation of the representational frame that is aligned with the body image is indispensable for the viewer to judge the left and right of the rotated body image from its own viewpoint. Therefore, the rotation of the body image can be considered to imply the rotation of the representational frame.

However, the multiprocess hypothesis differs from these two hypotheses in that it does not require the rotation of the body image. What is essential in the multiprocess hypothesis is the rotation of the representational frame, which enables the directional judgment from the mirror image's viewpoint. Although the multiprocess hypothesis does not exclude the possibility that the rotation of the body image may accompany that of the representational frame, this image rotation is not indispensable in the explanation of the Type I reversal proposed by the multiprocess hypothesis. Thus, this hypothesis is not susceptible to the criticism raised against the locomotion hypothesis and the symmetry hypothesis regarding why a mental image has to be superimposed.

Meanwhile, although the term rotation is used, this does not imply that the representational frame passes through the intermediate trajectory of the rotation, as in the case of mental rotation (Shepard \& Cooper, 1982; Shepard \& Metzler, 1971). What is needed in this explanation is only that the representational transformation locates the representational frame in the position where it is aligned with the mirror image's viewpoint, although the possibility of passing through the intermediate trajectory is not excluded.

\section{Social Learning Hypothesis}

Why does the viewer rotate the representational frame about a vertical axis and try to take the mirror image's viewpoint? Though two or more explanations are compatible with the multiprocess hypothesis, the most plausible one, which has never been proposed before, considers social learning a key factor.

To identify a part of a human body, the words left and right are often indispensable (e.g., "Raise your right hand."). This is because a human body is approximately bilaterally symmetrical and the symmetric parts have to be identified in terms of left and right (e.g., "the left eye" and "the right eye"). This is true for the global bilateral halves as well. These left and right are conventionally defined in reference to the orientational framework of the person who possesses that body. For instance, a person typically shakes hands with the "right" hand; this hand is located on the person's own right. If the orientational framework to judge right or left were not fixed in this way, it would be impossible to identify the symmetric body parts unambiguously.

Therefore, a viewer has to adjust his/her own orientational framework in accordance with another person's to identify correctly that person's body part that is indicated by the word left or right. When a viewer shakes hands with another person, for example, the viewer has to extend his/her own right hand toward the other person's hand that is on the right in reference to that person's orientational framework, not to the viewer's. When a surgeon conducts an operation on a patient's left lung, the surgeon has to open the chest that is on the left in reference to the patient's orientational framework; otherwise, the consequence would be disastrous for both of them.

In the typical encounter of two persons, as several researchers have pointed out, they face each other holding their upper bodies roughly upright. Accordingly, an appropriate transformation to assume the counterpart's orientational framework is typically a $180^{\circ}$ rotational transformation of a representational frame about a vertical axis. Everybody has to learn this transformation to have a normal social life by identifying other people's body parts correctly.

The social learning hypothesis assumes that a viewer applies the same learned rotation of a representational frame to the viewer's own mirror image as well to specify its symmetric parts by "left" or "right" in the conventional way. Before it is pointed out, most of us are unaware of the fact that we use different frames to refer to symmetric body parts, depending on whether they belong to ourselves or to others, while actually doing so. The rotation of a representational frame has become so automatic. Therefore, it is not surprising that this rotation is applied to the viewer's own mirror image as well.

The rotation about a vertical axis is not essential in itself. What is essential is to align the representational frame with the orientational framework of another person or one's own mirror image so that its body parts can be specified in the conventional way. Consider those body parts that are approximately symmetrical with respect to a horizontal axis (e.g., eyelids and lips). When a mother says, for example, "My child has lost one of his upper teeth," the lost tooth is the one that is closer to his nose, not the one that is closer to his neck, even if he is standing on his hands beside her. In this case, "up" is judged in reference to a representational frame that has been rotated about a horizontal axis, not a vertical axis, to be aligned with the child's body. 
Incidentally, the rotated representational frame can be used not only for identifying symmetric body parts but also for specifying a direction indicated by another person from his/her own viewpoint.

Once the reason for the transformation of a representational frame has been clarified, it is possible to better understand why this transformation has to be a rotation about a vertical axis when a viewer faces a mirror. To assume the own mirror image's viewpoint, the viewer has to first identify the body parts of the mirror image through a form recognition process. On the basis of the identified body parts, the viewer is able to set the up-down and front-back axes of a representational frame that is consistent with the mirror image's viewpoint: "Up" is the direction where the head is found, "front" is the direction where the eyes are found, and so on.

However, the left-right axis cannot be set on the basis of a particular organ because a human body is intrinsically symmetrical as far as its appearance is concerned. Although it is true that an individual body is not perfectly symmetrical, such an individual peculiarity cannot be relied on as a basis of identifying the left or right of a person in general. Nevertheless, it is not impossible to set the left-right axis unambiguously in the mirror image. In a Cartesian 3-D coordinate system, right is a direction that is $90^{\circ}$ clockwise from front when viewed from above. Since both front-back and up-down axes have already been located, it is possible to set the left-right axis on the basis of these two axes. When the viewer faces the mirror, the representational frame thus located results in a position that can be attained by rotating the viewer's own perceptual frame through $180^{\circ}$ about a vertical axis.

The social learning hypothesis resembles the symmetry hypothesis in referring to the approximate bilateral symmetry of a human body. However, the role of the approximate bilateral symmetry is quite different in the two hypotheses. In the symmetry hypothesis, the approximate symmetry is important because it minimizes discrepancy when a rotated body image is superimposed onto a mirror image. In the social learning hypothesis, the superimposition of the body image is not assumed. The approximate bilateral symmetry is important for two different reasons: (1) The symmetric body parts are conventionally identified in terms of left and right; and (2) the left and right of a person or its mirror image cannot be determined on the basis of its bilateral difference in shape.

It is true that the symmetry hypothesis also provides a fairly reasonable account of the rotation about a vertical axis in the Type I reversal. Although it has difficulty in explaining a left-right reversal in the mirror image of a character that is approximately symmetrical with respect to a horizontal axis (e.g., $C$ ) as pointed out earlier, this does not matter because this difficulty is concerned only with the Type II reversal (see the next section), whereas the present argument is confined to the Type I reversal.
However, the symmetry hypothesis cannot circumvent the other two difficulties mentioned previously. First, Navon (1987) maintained that a person with a congenitally asymmetric body will recognize a left-right mirror reversal, whereas the symmetry hypothesis makes a prediction to the contrary. The social learning hypothesis makes the same prediction as Navon's but for a different reason: The person with such a handicap still has to learn to identify others' symmetric body parts in terms of left and right. This learned convention may well be applied to this person's own mirror image. However, whether this kind of person actually recognizes such a mirror reversal or not is an empirical problem. Unfortunately, no reliable empirical evidence is available for the present, although Navon's factual judgment seems to be very plausible. Therefore, it is hard to decide unambiguously which hypothesis is preferred in this respect.

However, the superiority of the social learning hypothesis is clear with respect to the second pointnamely the reason for the rotation. The symmetry hypothesis has not provided a convincing account for why a viewer's body image has to be rotated to be superimposed onto his/her mirror image. As pointed out earlier, the superimposition is unnecessary according to recent research on form recognition. By contrast, the social learning hypothesis provides a convincing account for the rotation of a representational frame: In social life, there are many cases in which a person has to judge directions on the basis of a different person's orientational framework, especially for identifying that person's symmetric body parts.

\section{Type II Reversal}

This section will show how the multiprocess hypothesis explains the Type II reversal. In Figure $3 \mathrm{~A}$, a viewer sees an object's form directly and recognizes that its element $X$ is to the right of another element $I$ on the basis of the current frame. (In the case of the Type II reversal, a representational frame and a perceptual frame always coincide with each other. Accordingly, both of them will be referred to simply as a "frame.") To see the mirror, the viewer has to turn around: That is, the frame has to be rotated through $180^{\circ}$ on the vertical axis. In Figure 3B, the viewer sees the form's mirror image and recognizes that $X$ is to the left of $I$ on the basis of the rotated frame. To recognize the form of the mirror image (i.e., to specify its relation to stored cognitive representations), the viewer has to retrieve the cognitive representation of the real form on the basis of the rotated frame. (If the viewer keeps the representation activated while turning around, it can be conceived to be rotated together with the frame and the viewer's body.) In the representation, however, the viewer recognizes that $X$ is to the right of $I$ on the basis of the same frame (Figure 3B). By comparing the retrieved representation and the perceived mirror image, the viewer will recognize that left and right are 
A
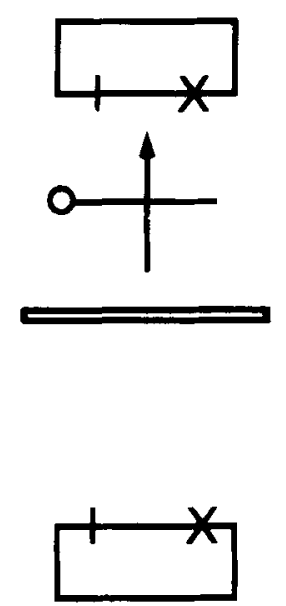

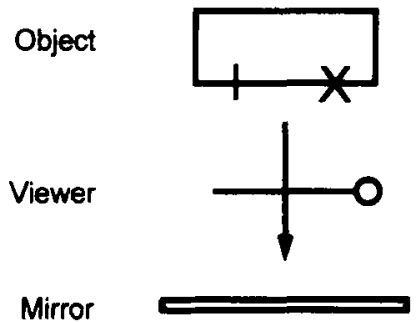

Mirror

image
B

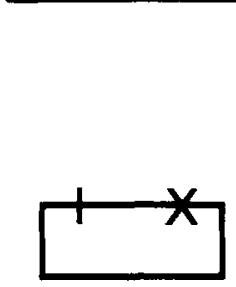

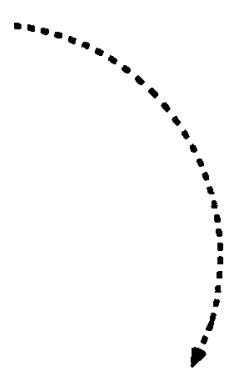

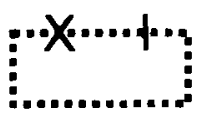

Representation

Figure 3. Type II reversal. The scene is sketched as it is viewed from above. A 3-D object as well as its mirror image is indicated by the rectangle drawn with solid lines. It carries a 2-D form on its surface facing the mirror. Two of the form's elements are indicated by $I$ and $X$. The retrieved representation of the object is indicated by the rectangle drawn with dotted lines. The viewer is indicated by the cross, which faces in the direction indicated by the arrow, and supposed to have a watch, which is indicated by a circle, around the left wrist. (A) At the beginning, the viewer faces the object to see the form directly, in which $X$ is to the right of $I$. (B) The viewer turns around to see the object's mirror image, in which $X$ is to the left of $I$. However, $X$ is to the right of $I$ in the retrieved representation. When the viewer compares the mirror image with the representation, a left-right reversal is recognized.

reversed between them. The Type II reversal is thus explained.

It is the $180^{\circ}$ rotation of the frame on a vertical axis that has caused the left-right reversal. This rotation will be referred to as a "physical transformation" because it is produced by the rotational transformation of the viewer's body. Although the transformation of the body passes through the intermediate trajectory of the rotation, it is not indispensable for the transformation of the representation to do so. If the viewer deactivates the representation before the rotation and retrieves it after the rotation, it does not pass through the intermediate trajectory though the same consequence is obtained. Furthermore, the representation does not have to be identified with a mental image. It may take a more abstract form (Pylyshyn, 1973, 1984), although the possibility that it takes a form of a mental image is not excluded.

To explain the Type II reversal precisely, the optical transformation by the mirror has to be taken into account. Table 2 shows how the combination of the optical and physical transformations produces a left-right mirror reversal. When the viewer faces the real object, the directions are ascribed to it by the current frame (i.e., the first frame; see the Real object $\times$ First cell). The object's representation (i.e., the first representation) is constructed on the basis of the first frame (see the Representation $\times$ First cell). When the object is reflected in the mirror, the optical transformation reverses the frontback direction (see the Mirror image $\times$ First cell).

To face the mirror, the viewer turns around and the first frame is converted into the second frame. This physical transformation reverses both front-back and leftright axes between the first and second frames. As noted earlier, these consequences can be explained in terms of elementary geometry. After turning around, the viewer identifies the figural elements of the mirror image through a form recognition process. When the viewer judges their directions on the basis of the second frame,

Table 2

Type II Reversal

\begin{tabular}{lccc}
\hline & \multicolumn{3}{c}{ Frame } \\
\cline { 2 - 4 } Form & First & \multicolumn{1}{c}{ Second } \\
\hline Representation & $\mathrm{U}-\mathrm{D} / \mathrm{F}-\mathrm{B} / \mathrm{L}-\mathrm{R}$ & $\rightarrow$ & $\mathrm{U}-\mathrm{D} / \mathrm{F}-\mathrm{B} / \mathrm{L}-\mathrm{R}$ \\
Real object & $\mathrm{U}-\mathrm{D} / \mathrm{F}-\mathrm{B} / \mathrm{L}-\mathrm{R}$ & & \\
Mirror image & $\mathrm{U}-\mathrm{D} / B-F / \mathrm{L}-\mathrm{R}$ & $\rightarrow$ & $\mathrm{U}-\mathrm{D} / \mathrm{F}-\mathrm{B} / \mathrm{R}-\mathrm{L}$ \\
Comparison & & & $(--) /(-) /(\mathrm{R})$ \\
\hline
\end{tabular}

Note-First, the frame to see the real object; Second, the frame to see the mirror image. The uppercase letters indicate the directions first ascribed to the real object by the first frame: U-D, up-down; F-B, front-back; $\mathrm{L}-\mathrm{R}$, left-right. The reversal of each direction is indicated by reversing an order (e.g., L-R vs. $R-L)$. (R), reversal; (-), nonreversal in the final comparison. 
only the left-right direction is reversed because the reversal of the front-back axis is canceled out by the reversal due to the optical transformation (see the Mirror image $\times$ Second cell).

The viewer retrieves the representation of the object (i.e., the second representation) in this position. Its directions judged by the second frame remain unchanged (see the Representation $\times$ Second cell) because the representation has been reconstructed on the basis of this frame. When the viewer compares the directions of the mirror image with those of the representation on the basis of the second frame, therefore, only the left-right direction is reversed (see the Comparison $\times$ Second cell). The Type II reversal is thus explained with the optical transformation taken into account. Again, the leftright mirror reversal occurs precisely because the mirror does not reverse left and right.

The absence of a front--back reversal usually goes unnoticed when a 2-D form like an alphanumeric character is seen. However, a viewer could easily recognize this absence while looking at a 3-D form. Suppose, for example, that the object indicated by a rectangle in Figure 3 is a clock. When the viewer looks at the mirror image, the clock's face is reversed regarding left and right. In addition, the face is closer to the viewer than its rear side (see Figure $3 \mathrm{~B}$ ) just as in the real clock (Figure $3 \mathrm{~A}$ ) or in its representation (Figure $3 \mathrm{~B}$ ). The nonreversal of front and back is apparent.

The reason that the physical transformation is conducted on a vertical axis is that a viewer usually turns on a vertical axis to see a mirror. The explanation by the multiprocess hypothesis resembles the one by the locomotion hypothesis in this respect. However, there are two critical differences. First, what is rotated is the mental image of the viewer's body in the locomotion hypothesis, whereas it is the viewer's real body in the multiprocess hypothesis. Second, unlike the locomotion hypothesis, the multiprocess hypothesis does not assume that the rotation is always conducted on a vertical axis; it simply asserts that a left-right reversal is recognized when the rotation is conducted on a vertical axis. If the rotation is conducted on a horizontal axis, the multiprocess hypothesis predicts that an up-down reversal will be recognized instead of a left-right one. In the case of the rotation on a horizontal axis, the front-back and up-down axes are reversed by the physical transformation. When the viewer compares the mirror image with the second representation, only the up-down reversal is recognized because the front-back direction is further reversed by the mirror. Suppose, for example, that a viewer looks at the mirror image of a character from between the legs holding the head upside down. The character will look reversed regarding up and down but not reversed regarding left and right. The mirror reversal is usually identified with a left-right reversal simply because people usually turn around on a vertical axis to see a mirror.
As long as the left-right direction is reversed between a mirror image and its corresponding cognitive representation, any setting could produce a left-right mirror reversal. For example, the rotation of an object could substitute for that of a viewer's body when the object can be rotated at all. Suppose that both a mirror and a character on a sheet of paper face a viewer at the beginning; the character is not reflected in the mirror at this moment. Suppose further that the viewer rotates the paper through $180^{\circ}$ on a vertical axis. Then the viewer will see the character's mirror image, which is reversed with respect to left and right. In this case, the viewer's body is not rotated. However, the rotation of the paper ends up with the same consequence as that obtained after the rotation of the viewer's body in Figure 3: After the rotation, the left and right of the mirror image and those of the representation are reversed in relation to each other. Therefore, the rotation of the object is functionally equivalent to that of the viewer's body.

The actual rotation of a physical entity, whether a body or an object, may even be lacking altogether. As pointed out earlier in a criticism against the rotation hypothesis, a viewer recognizes the left-right reversal of an alphanumeric character in a rearview mirror even if the viewer has never seen its original. In this case, the viewer rotates neither his/her own body nor the character. How is the mirror reversal produced? The retrieved representation and its original have the prototypical form of that character in common. Though they may be different in details, this difference is irrelevant to the recognition of the character. The mirror image also shares this prototypical form except for the reversal concerning left and right. The viewer retrieves the representation that has the same prototypical form on the basis of the perceived elements of the mirror image. When the viewer compares this representation with the mirror image, therefore, the viewer recognizes that the mirror image is reversed with regard to left and right. To sum up, the essence of the Type II reversal is not the rotation of a physical entity, but this directional discrepancy between a mirror image and its corresponding cognitive representation.

This explanation of the Type II reversal by the multiprocess hypothesis resembles the one offered by the rotation hypothesis. However, the present hypothesis is preferred in that it is not susceptible to the criticisms raised against the rotation hypothesis. First, the Type I reversal has been correctly excluded from the scope of the same underlying principle. As a result, the criticism that the mirror reversal of a viewer's own body cannot be explained does not apply to the present hypothesis. Second, the optical transformation by a mirror has been properly taken into account. As a result, the present hypothesis is not susceptible to the criticism that the rotation of an object shows its rear side and a front-back reversal. Finally, and most importantly, the underlying principle of the Type II reversal has been identified explicitly. The rotation hypothesis left the following ques- 
tions unanswered: What is the relation between the rotation of an object and that of a viewer? Between what and what is a mirror reversal recognized? By identifying the underlying principle, the present hypothesis has succeeded in providing reasonable answers for these questions. In addition, the multiprocess hypothesis has succeeded in explaining a mirror reversal recognized when neither an object nor a viewer is rotated.

In the meantime, Morris (1993) criticized the rotation hypothesis because it cannot explain a left-right reversal of an upside down person. In the left panel of Figure 1 in Morris, both an upright body and an upside down body are facing the reader together with upright characters and upside down characters, respectively. According to the rotation hypothesis, the appearance of an object's mirror image is identical to that of the rotated object. It thus predicts that only up and down will be reversed when the object is rotated about a horizontal axis, as in this figure. The multiprocess hypothesis makes the same prediction in this respect. Morris admitted that this prediction holds true for the characters. However, he claimed that the left and right are also reversed in the case of the bodies. In his Figure 1, the upright body has a watch around the left wrist, which is indicated by "figure's left arm," whereas the upside down body has a watch around the right wrist, which is indicated by "figure's right arm." Morris called this discrepancy between the characters and bodies a "paradox" (p. 874).

However, there is no real paradox. In his Figure 1, both bodies face the reader, and the two watches are both on the right if judged from the reader's viewpoint. Accordingly, the bodies and characters must be drawn on a transparent sheet. Otherwise, the upside down bodies and characters would not look as they do in this figure. Then, consider what orientational frameworks Morris (1993) used to judge the respective reversals.

In the case of the characters, he must have applied his own (i.e., the reader's) viewpoint consistently to both upright and upside down characters in judging that the up and down were reversed whereas the left and right were not. In judging that the up and down were reversed between the upright and upside down bodies, he must have also applied an identical (i.e., the reader's) viewpoint consistently to both bodies. In judging that the left and right were reversed between those bodies, however, he must have used the upright body's viewpoint to judge its own left and right, whereas he must have used the upside down body's viewpoint to judge its own left and right. That is, the "paradox" originated from this inconsistency in employing the orientational frameworks. If Morris (1993) had consistently applied an identical viewpoint to both bodies in judging their left and right, he would have recognized no left-right reversal. Alternatively, if he had not failed to adopt the upside down body's viewpoint in judging its own up and down, he would have recognized no up-down reversal. Thus, his criticism invalidates neither the rotation hypothesis nor the multiprocess hypothesis. Incidentally, this "paradox" attests to the importance of specifying orientational frameworks appropriately in discussing various issues concerning the mirror reversal.

The social learning hypothesis provides a reasonable account for this common failure to employ an upside down representational frame in judging the up and down of an upside down human body: We have learned to rotate a representational frame to identify symmetric body parts that are conventionally identified in terms of left and right. As a consequence, we automatically base our judgments about the left and right of a human body on this rotated representational frame. However, we have not been trained to judge the up and down of a whole human body on the basis of an upside down representational frame because a human body as a whole is not symmetric with respect to a horizontal axis, and its parts (e.g., a head vs. feet) are not conventionally identified by "up" and "down." Thus, we do not automatically base our judgments about the up and down of a whole human body on the upside down representational frame when the body is inverted.

In judging up and down, we usually rely on an environmental up - down axis, which is determined by terrestrial gravitation, the sky and ground, and so on. This axis is reliable because it is common to all neighboring people and remains identical irrespective of their posture, though it cannot be used to determine left-right or frontback. When we judge the up and down of an upside down human body, too, we base our judgment on this environmental up-down axis. As a result, we recognize an updown reversal. The employment of the environmental axis has become so automatic that Morris (1993) must have judged the up and down of the upside down body in reference to this axis and recognized the illusory paradox.

Earlier in this section, it was pointed out that an updown reversal will be recognized when a viewer looks at the mirror image of a character from between the legs holding the head upside down. In this setting, we tend to overlook the predicted up-down reversal. This failure can be explained easily if it is taken into account that we usually base our up-down judgment on the environmental up-down axis rather than our own upside down head.

Although the reliance on the environmental axis is typical, there are exceptions. As noted earlier, we use a representational frame aligned with an inverted body to identify its parts that are symmetrical with respect to a horizontal axis (e.g., teeth, eyelids, and so on). We do not apply this convention to a whole body because its global shape is not symmetrical with respect to a horizontal axis, although it is bilaterally symmetrical. The social learning hypothesis is thus consistent with whether we employ the environmental axis or a rotated representational frame in judging the up and down of a human body.

\section{Differences Between Type I and II Reversals}

When the distinction between the Type I and Type II reversals was first introduced, the Type I was related to the mirror image of a viewer's own body, whereas the 
Type II was related to that of an alphanumeric character. Strictly speaking, however, the distinction between these two types of reversal cannot be defined in terms of the kinds of reflected objects.

Suppose, for example, that a pedestrian sees a mirror at a crossroads, which reflects a road that extends to the pedestrian's right and an automobile that shows its front on this road. Suppose further that the pedestrian sees the automobile's steering wheel on the right behind the windshield. Is it a car with a right-hand drive or a left-hand drive? We tend to judge that this is a left-hand-drive. This judgment is wrong; it is actually a right-hand-drive. We make such an error because we automatically rotate our representational frame to assume the automobile's viewpoint. Therefore, this left-right reversal has to be classified as a Type I reversal although an automobile, not a human body, is concerned. Generally speaking, the rotation of a representational frame may be conducted for those objects that are approximately bilaterally symmetrical and the parts of which are usually identified in terms of left and right (e.g., animals, airplanes, and so on).

Conversely, some left-right mirror reversals of a human body may be classified as Type II reversals. Suppose that a person with a watch around the left wrist is facing a mirror. When a viewer faces this object person directly, the watch is on the right if judged from the viewer's perceptual frame. Then, the viewer turns around to see the mirror. In the mirror image, the watch is on the left if judged by the viewer's perceptual frame. If the viewer compares the mirror image with the representation of the real object person on the basis of the perceptual frame, therefore, a left-right reversal will be recognized. Although a human body is concerned, this reversal has to be classified as a Type II reversal because the representational frame is not rotated away from the perceptual frame. ${ }^{1}$

Thus, the distinction between the two types of reversal cannot be made in terms of the kinds of reflected objects. It has to be defined in terms of the underlying principles. The Type I reversal emerges from the comparison between a real object and its mirror image. The directions of the real object are judged in reference to the perceptual frame, whereas those of the mirror image are judged in reference to the representational frame aligned with it. The reason for the left-right reversal is that this representational frame is set in the position that can be attained by rotating the perceptual frame through $180^{\circ}$ about a vertical axis. The Type II reversal emerges from the comparison between the representation of an object and its mirror image. The directions of the mirror image and those of the representation are judged in reference to the perceptual frame and the representational frame that coincides with the perceptual frame, respectively. The reason for the reversal is that the representation is retrieved in the position that can be attained by rotating the real object through $180^{\circ}$ about a vertical axis.

\section{Type III-I Reversal}

A viewer recognizes a left-right reversal while standing "shoulder to a mirror." Block (1974) highlighted this
Table 3 Type III-I Reversal

\begin{tabular}{lcrr}
\hline & \multicolumn{2}{c}{ Frame } \\
\cline { 2 - 3 } Form & Perceptual & & Representational \\
\hline Real object & $\mathrm{U}-\mathrm{D} / \mathrm{F}-\mathrm{B} / \mathrm{L}-\mathrm{R}$ & & \\
Mirror image & $\mathrm{U}-\mathrm{D} / \mathrm{F}-\mathrm{B} / R-L$ & $\rightarrow$ & $\mathrm{U}-\mathrm{D} / \mathrm{F}-\mathrm{B} / R-L$ \\
Comparison & & & $(-) /(-) /(\mathrm{R})$ \\
\hline
\end{tabular}

Note-The uppercase letters indicate the directions first ascribed to the real object by the perceptual frame: $U-D$, up-down; F-B, front-back; $\mathrm{L}-\mathrm{R}$, left-right. The reversal of each direction is indicated by reversing an order (e.g., L-R vs. $R-L)$. (R), reversal; (-), nonreversal in the final comparison.

case in criticizing the conventional description hypothesis. This reversal is produced by a principle that is entirely different from those underlying Type I and Type II reversals.

As usual, the optical transformation produces a reversal along an axis perpendicular to the mirror's surface, which coincides with a left-right direction in this case. Therefore, the optical transformation causes a left-right reversal (see the Mirror image $\times$ Perceptual cell in Table 3). A representational transformation needed to take the mirror image's viewpoint is not a rotation but a translation along the left-right axis in this case. This transformation produces no reversal on any axis (see the Mirror image $\times$ Representational cell). When the viewer judges the directions of the mirror image on the basis of this translated representational frame, a left-right reversal is recognized (see the Comparison $\times$ Representational cell). In this case, it is the mirror that produces the left-right reversal. Researchers called this reversal a "true" reversal (Bennett, 1970; Block, 1974; Gardner, 1964,1990 ) probably because it is produced optically by the mirror. At any rate, the principle underlying this reversal is entirely different from those underlying Type I and Type II reversals. Therefore, it has to be classified into a separate category.

\section{Type III-II Reversal}

Such an optically created reversal is not confined to a human body, of course. Suppose, for example, that the viewer who is standing "shoulder to the mirror" is holding in front of himself/herself a sheet of paper carrying a character so that it is perpendicular to the mirror. The

Table 4

Type III-II Reversal

\begin{tabular}{lc}
\multicolumn{1}{c}{ Form } & Frame \\
\hline Representation & $\mathrm{U}-\mathrm{D} / \mathrm{F}-\mathrm{B} / \mathrm{L}-\mathrm{R}$ \\
Real object & $\mathrm{U}-\mathrm{D} / \mathrm{F}-\mathrm{B} / \mathrm{L}-\mathrm{R}$ \\
Mirror image & $\mathrm{U}-\mathrm{D} / \mathrm{F}-\mathrm{B} / \mathrm{R}-L$ \\
Comparison & $(-) /(-\mathrm{-}) /(\mathrm{R})$ \\
\hline
\end{tabular}

Note-The uppercase letters indicate the directions first ascribed to the real object: U-D, up-down; F-B, front-back; L-R, left-right. The reversal of each direction is indicated by reversing an order (e.g., $L-R$ vs. $R-L)$. (R), reversal; $(-)$, nonreversal in the final comparison. 
viewer will recognize that the character reflected in the mirror is reversed with respect to left and right.

The process of this left-right reversal is shown in Table 4, where both perceptual and representational frames are simply referred to as "Frame." As in the case of a human body, the left-right direction is optically reversed by the mirror (see the Mirror image $\times$ Frame cell). The representational frame does not have to be translated because what is reflected is not a human body but a character. Although the mirror image can be compared with the representation of the character, it can also be compared directly with the real character because the latter is also visible in this layout. In either comparison, the left-right direction is reversed (see the Comparison $\times$ Frame cell).

This left-right reversal is slightly different from the reversal of the viewer's own mirror image because the translation of the representational frame is not involved, although the reversal itself is also caused by the mirror's optical transformation. Therefore, the optical left-right reversal of a character should be classified into the subcategory Type III-II, and that of a viewer should be classified into the subcategory Type III-I.

The principle of the Type III-II reversal also underlies the mirror reversal of a character above a mirror. When a sheet of paper carrying an alphanumeric character is held vertical above a mirror that is put on a horizontal surface, the mirror image of the character shows an up-down reversal instead of a left-right one. This phenomenon was formerly mentioned as evidence against the conventional description hypothesis and the symmetry hypothesis. In this case, the up-down direction is perpendicular to the mirror and thus reversed optically by the mirror. This is why the up-down reversal is recognized. No physical transformation is needed to see the mirror image because both the real character and its mirror image can be seen at the same time in this layout, and thus the viewer does not have to turn around to see the mirror image. Since it is the physical transformation that is responsible for the usual (Type II) left-right reversal, no left-right reversal is produced in this case.

One might argue that this mirror image actually contains a left-right reversal because its $180^{\circ}$ mental rotation in the picture plane makes it clear that this mirror image is the enantiomorph of the original character. As an illustration, a lowercase letter $b$ becomes $p$ in the mirror underneath it; and $p$ rotated through $180^{\circ}$ on the page becomes $d$, which is the enantiomorph of $b$. However, it is not the mirror but the mental rotation that produces the left-right reversal in this case. If all forms that become left-right reversed after a $180^{\circ}$ mental rotation had to be considered as left-right reversed, all the normal alphanumeric characters would also have to be considered as left-right reversed because they become left-right reversed after a $180^{\circ}$ mental rotation. Obviously, there would be no basis to distinguish between left-right reversal and nonreversal. Therefore, the above argument is not justified.
It is not accurate to define the difference between a form and its enantiomorph in terms of a left-right reversal alone. It is true that the orientational relationships with respect to left and right in the enantiomorph are reversed when compared with those in the original if they are both upright (i.e., their orientational relationships with respect to up and down are identical). However, there will be no difference with respect to left and right when one of them is inverted (i.e., their orientational relationships with respect to up and down are reversed). Accordingly, both left-right and up-down have to be taken into consideration to define the enantiomorph of a 2-D form in reference to a Cartesian coordinate system.

\section{THE ACCOUNTS OF OTHER DISPUTED ISSUES}

The preceding section has shown how the multiprocess hypothesis explains the basic mirror reversal phenomena of Types I, II, and III. This section will show how the hypothesis explains those phenomena that have been presented as evidence against the past hypotheses.

\section{The Case of a Mirror on a Ceiling or a Floor}

A viewer recognizes a left-right reversal while looking at the mirror image of his/her own body in a mirror on a ceiling or a floor. This reversal was presented as evidence against the locomotion hypothesis and the frontal encounter schema hypothesis. Why does the viewer recognize this reversal?

The optical transformation produces a reversal along the axis perpendicular to its surface, as usual, but it results in the reversal of the up-down direction because this direction is perpendicular to the mirror in this case. The viewer performs the representational transformation as a rotation of a representational frame about a frontback axis that is on the mirror's surface because it is an appropriate transformation to take the mirror image's viewpoint. This transformation reverses both up-down and left-right axes of the representational frame. However, the already reversed up-down direction of the mirror image is judged to be normal by this reversed updown axis. Accordingly, the viewer recognizes only a left-right reversal in judging the directions of the mirror image on the basis of this rotated representational frame.

In this case, we often entertain the following doubt: The body image in the mirror on the ceiling or floor actually looks upside down although the multiprocess hypothesis seems to predict that it should be recognized as upright. As discussed earlier (see the Type II Reversal section), we usually judge the up and down of an inverted human body on the basis of the environmental up-down axis instead of the representational frame aligned with the inverted body; thus the body image in the mirror on the ceiling or floor looks upside down. The inverted up-down axis of the rotated representational frame is used only to set its left-right axis (see the Social Learning Hypothesis section). 


\section{The Case of Lying Down}

When a viewer faces an upright mirror while lying down on a floor, the viewer still recognizes a left-right reversal. Navon (1987) referred to this case as evidence against the locomotion hypothesis.

The solution offered by the multiprocess hypothesis is straightforward: The viewer has to rotate the representational frame about a horizontal axis through $180^{\circ}$ to take the mirror image's viewpoint. Therefore, the transformations in Table 1 are conducted and then the left-right reversal is recognized. The only differences between the case of lying down and the case of standing are that the up-down direction is horizontal in the former case whereas it is vertical in the latter case, and that the left-right direction is vertical in the former case whereas it is horizontal in the latter case. However, these differences have nothing to do with the recognition of the mirror reversal.

\section{The Case of a Concave Cylinder Mirror}

Some researchers have pointed out that a left-right reversal is not observed in a concave cylinder mirror (e.g., Block, 1974; Gardner, 1964, 1990; Navon, 1987). This case can be taken as evidence against the conventional description hypothesis and the symmetry hypothesis. Suppose that an alphanumeric character written on a sheet of paper is reflected in the inside of a concave cylinder mirror that is placed so that its axis is vertical. Such a mirror has a focal line where the light reflected from its left meets that reflected from its right. When the sheet and the viewer are both located farther away from the mirror than its focal line, the viewer will see a normal character instead of an ordinary mirror image with a left-right reversal.
It is a characteristic peculiar to the concave cylinder mirror that is responsible for this absence of a left-right reversal. The light reflected by the left of the mirror and that reflected by its right interchange after passing the focal line. When seen from a point farther away than the focal line, therefore, this mirror optically causes a reversal with respect to left and right (Thomas, 1980) in addition to the one with respect to front and back. A normal character is seen in the mirror because both leftright and front-back reversals caused by the rotation of the character or viewer are canceled out by these optical reversals of both left-right and front-back directions. The image in the concave cylinder mirror is not reversed with respect to left and right precisely because it does reverse left and right.

An essentially identical explanation applies to a viewer's own body as well. The left-right reversal due to the $180^{\circ}$ rotation of a representational frame about a vertical axis is canceled out by the optical left-right reversal due to the concave cylinder mirror. Thus, no left-right reversal is recognized in the mirror image of the viewer, either.

\section{The Case of Two Mirrors}

Suppose that two mirrors are set so that they are roughly perpendicular to each other (see Figure 4). Navon (1987) referred to this case as evidence against the conventional description hypothesis (p. 279); it contradicts the symmetry hypothesis as well. This case is especially interesting because the separation of Types I and II is directly seen. In the horizontal mirror that reflects the vertical mirror, an alphanumeric character is reversed with regard to left and right, whereas a viewer's own body is not. Of course, the mirror does not discriminate between a character and a body. So, why is this difference produced?
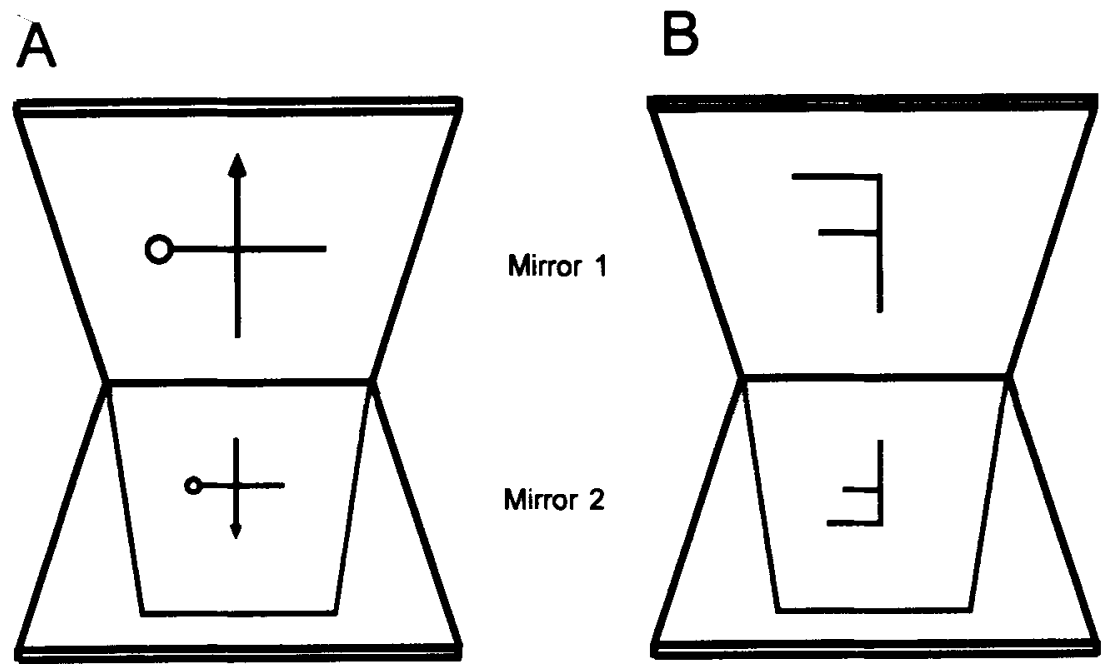

Figure 4. The cases of two mirrors. A real viewer is omitted in either panel. (A) A viewer who has a watch around the left wrist faces Mirror 1; his/her mirror image is further reflected in Mirror 2. The arrow indicates the direction, up, not front, in this figure; the frontback axis is omitted, and the watch is indicated by the circle. (B) An uppercase letter, F, faces Mirror 1; its mirror image is further reflected in Mirror 2. Though the mirnor image first reflected in Mirror 2 is further reflected in Mirror 1, it is omitted in both panels. 
Table 5

The Case of Two Mirrors Reflecting a Viewer's Body

\begin{tabular}{|c|c|c|c|}
\hline \multirow[b]{2}{*}{ Form } & \multicolumn{3}{|c|}{ Frame } \\
\hline & Perceptual & & Representational \\
\hline Real object & $\underset{\downarrow}{\mathrm{U}-\mathrm{D} / \mathrm{F}-\mathrm{B} / \mathrm{L}-\mathrm{R}}$ & & \\
\hline Mirror image 1 & $\underset{\downarrow}{\mathrm{U}-\mathrm{D} / B-F / \mathrm{L}-\mathrm{R}}$ & & \\
\hline Mirror image 2 & $D-U / B-F / \mathrm{L}-\mathrm{R}$ & $\rightarrow$ & $\mathrm{U}-\mathrm{D} / \mathrm{F}-\mathrm{B} / \mathrm{L}-\mathrm{R}$ \\
\hline Comparison & & & $(-) /(-) /(-)$ \\
\hline
\end{tabular}

Note-The uppercase letters indicate the directions first ascribed to the real object by the perceptual frame: $U-D$, up-down; F-B, front-back, $\mathrm{L}-\mathrm{R}$, left-right. The reversal of each direction is indicated by reversing an order (e.g., U-D vs. $D-U)$. (-), nonreversal in the final comparison.

In Figure 4A, the viewer's body is first reflected in Mirror 1; this mirror image is further reflected in Mirror 2 . When the viewer looks at the latter mirror image in Mirror 2, the viewer finds no left-right reversal. (In Figure 4, the real viewer who is supposed to face Mirror 1 is not shown. Note that the direction "up," not "front," of the viewer is indicated by the arrow in Figure 4A. Although the mirror image that is given as a result of reflecting the mirror image first reflected in Mirror 2 can be seen in Mirror 1, it is omitted to avoid confusion.)

Table 5 shows why no reversal is recognized. Initially, the directions are ascribed to the viewer's real body on the basis of the perceptual frame (see the Real object $X$ Perceptual cell). When the body is reflected in Mirror 1, the front-back direction of the body is reversed, as usual, by the optical transformation (see the Mirror image $1 \times$ Perceptual cell). When the mirror image in Mirror 1 is further reflected in Mirror 2, the up-down direction is reversed by the optical transformation because this direction is perpendicular to Mirror 2. In Mirror 2, therefore, both up-down and front-back directions are reversed (see the Mirror image $2 \times$ Perceptual cell). To take the second mirror image's viewpoint, finally, the viewer has to rotate the representational frame alone through $180^{\circ}$ about a horizontal axis, leaving the perceptual frame in the real body. This representational transformation reverses both up-down and front-back axes. As a result, the reversals of the up-down and frontback directions of the second mirror image are both canceled out when judged by this rotated representational frame (see the Mirror image $2 \times$ Representational cell). Throughout the above transformations, the left-right direction has never been reversed. Therefore, when the viewer compares the directions of the second mirror image in Mirror 2 judged by the rotated representational frame to the initial directions ascribed to the real body by the perceptual frame, no reversal whatsoever is recognized (see the Comparison $\times$ Representational cell).

Incidentally, the viewer often recognizes an up-down reversal in this case. This is because we usually judge the up-down of an inverted human body on the basis of the environmental up-down axis (see the Type II Reversal section).
When the viewer looks at the mirror image of an alphanumeric character in Mirror 2, however, the viewer recognizes both left-right and up-down reversals. (See Figure 4B: In this figure, both real viewer and real character are omitted; they are both supposed to face Mirror 1 . Although the mirror image that is given as a result of reflecting the mirror image first reflected in Mirror 2 can also be seen in Mirror 1, it is again omitted to avoid confusion.)

Table 6 shows how these reversals are recognized. To see the real character, the viewer has to be initially facing it between it and Mirror 1. In this position, the viewer constructs its representation on the basis of the initial frame. (The term frame is used because the representational frame always coincides with the perceptual frame in this case.) The directions are ascribed to the real character and its representation on the basis of this first frame (see the Real object $\times$ First and Representation $\times$ First cells). Then, the viewer rotates about a vertical axis through $180^{\circ}$ to face Mirror 1 . This physical transformation reverses both front-back and left-right axes of the frame. Accordingly, the corresponding directions of the real character are reversed (though it is invisible) if judged by the second frame (see the Real object $\times$ Second cell). When the real character is reflected in Mirror 1 , this optical transformation reverses the front-back direction, as usual. ${ }^{2}$ As a result, its former reversal is canceled out (see the Mirror image $1 \times$ Second cell). When the character's mirror image in Mirror 1 is further reflected in Mirror 2, this second optical transformation reverses the up-down direction because it is perpendicular to Mirror 2 (see the Mirror image $2 \times$ Second cell). When the viewer compares the directions of the representation with those of the second mirror image on the basis of the second frame, the up-down and left-right directions are reversed, whereas the front-back direction is not (see the Comparison $\times$ Second cell). Thus, the viewer recognizes both left-right and up-down reversals.

In the case of the character, the left-right reversal is produced by the $180^{\circ}$ physical transformation about a

Table 6

The Case of Two Mirrors Reflecting a Character

\begin{tabular}{|c|c|c|c|}
\hline \multirow[b]{2}{*}{ Form } & \multicolumn{3}{|c|}{ Frame } \\
\hline & First & & Second \\
\hline Representation & $\begin{array}{c}\mathrm{U}-\mathrm{D} / \mathrm{F}-\mathrm{B} / \mathrm{L}-\mathrm{R} \\
\uparrow\end{array}$ & $\rightarrow$ & $\mathrm{U}-\mathrm{D} / \mathrm{F}-\mathrm{B} / \mathrm{L}-\mathrm{R}$ \\
\hline Real object & $\mathrm{U}-\mathrm{D} / \mathrm{F}-\mathrm{B} / \mathrm{L}-\mathrm{R}$ & $\rightarrow$ & $\begin{array}{c}\mathrm{U}-\mathrm{D} / B-F / R-L \\
\downarrow\end{array}$ \\
\hline Mirror image 1 & & & $\begin{array}{c}\mathrm{U}-\mathrm{D} / \mathrm{F}-\mathrm{B} / R-L \\
\downarrow\end{array}$ \\
\hline Mirror image 2 & & & $D-U / \mathbf{F}-\mathbf{B} / R-L$ \\
\hline Comparison & & & $(\mathrm{R}) /(-) /(\mathrm{R})$ \\
\hline
\end{tabular}

Note-First, the frame to see the real character. Second, the frame to see the mirror image. The uppercase letters indicate the directions first ascribed to the real object by the first frame: $\mathrm{U}-\mathrm{D}$, up-down; F-B, front-back; $L-R$, left-right. The reversal of each direction is indicated by reversing an order (e.g., $\mathbf{L}-\mathrm{R}$ vs. $R-L)$. (R), reversal; $(-)$, nonreversal in the final comparison 
vertical axis, as in the typical Type II reversal. In the case of the viewer's own body, however, a $180^{\circ}$ rotation of the representational frame about a vertical axis, which produces a left-right reversal in the typical Type I reversal, is never conducted. Accordingly, a left-right reversal is not recognized in Mirror 2 regarding the viewer's own body. Thus, the discrepancy between Types I and II becomes apparent in the case of the two mirrors.

\section{CONCLUSIONS}

The multiprocess hypothesis proposes that the mirror reversal, which has been considered to be a single phenomenon, should be divided into three different phenomena that result from different underlying principles-Types I, II, and III-which is further divided into two subcategories-Types III-I and III-II.

The Type I reversal is primarily related to a viewer's own body, whereas the Type II is primarily related to alphanumeric characters. However, they cannot be defined strictly in terms of the kinds of reflected objects; a more rigorous distinction has to be made in terms of their underlying principles.

To understand the Type I reversal correctly, two kinds of orientational frameworks have to be distinguished: (1) a perceptual frame, which is used for perceptual encoding and is always congruent with a viewer's body; and (2) a representational frame, which is used for constructing and processing spatial representations and can be separated from a viewer's body. The Type I reversal emerges from the comparison between the orientational relationships in a real object judged by a perceptual frame, on the one hand, and those in its mirror image judged by a representational frame that is congruent with the mirror image's viewpoint, on the other. The primary cause for the ordinary left-right reversal is twofold: (1) A plane mirror optically reverses only front and back, leaving left and right unchanged; and (2) the representational frame is set in the position that could be attained if the perceptual frame were rotated through $180^{\circ}$ about a vertical axis. The social learning hypothesis proposes that the representational frame is set in this position primarily to specify symmetric body parts of the mirror image in the conventional way from its own viewpoint.

The Type II reversal emerges from the comparison between the orientational relationships in an object's mirror image judged by a frame that is congruent with a viewer's body, on the one hand, and those in the object's mental representation judged by the same frame, on the other. The primary cause of the ordinary left-right reversal is again twofold: (1) A plane mirror optically reverses only front and back, leaving left and right unchanged; and (2) the mental representation is retrieved in the position that could be attained if the real object were rotated through $180^{\circ}$ about a vertical axis.

The Type III reversal emerges from the comparison between the orientational relationships in a real object or its mental representation, judged by a frame that is con- gruent with a viewer's body, on the one hand, and those in its mirror image, on the other. The Type III is further divided into the Types III-I and III-II, depending on whether the orientational relationships in the mirror image are judged by the perceptual frame or a translated representational frame. In both subtypes, however, the primary cause of the reversal is identical--namely, the optical transformation by the mirror.

In each type of reversal, the specified underlying principle provides a reasonable account for an up-down reversal or a nonreversal observed in other settings. As confirmed below, furthermore, the multiprocess hypothesis is free from all the criticisms raised against the preceding hypotheses.

One of Morris's (1993) criticisms of Haig's (1993) optical hypothesis concerns the reason why a viewer has to take the hypothetical viewpoint behind the mirror ( $F$ in Figure 1), which is not equivalent to the actual viewpoint ( $E$ in Figure 1 ) as to the very judgment about left and right. In the account of the Type I reversal by the multiprocess hypothesis, the reason for taking the mirror image's viewpoint has been made explicit: That is, it is taken to specify the body parts in the conventional way. The other criticism is that optics does not provide a reasonable account as to why the viewpoint has to be rotated about a vertical axis, not about a horizontal axis. The multiprocess hypothesis is unambiguous as to how a representational frame is transformed: It is determined through a form recognition process applied to the concerned mirror image.

The reason why a pencil aligned with the vertical line $\mathrm{AC}$ or the horizontal line BD (see Figure 1) does not look reversed in the mirror is readily comprehensible within the theoretical framework of the multiprocess hypothesis. We do not have to assume the mirror image's viewpoint in the case of a pencil; its left and right are not perpendicular to the mirror. Therefore, this case has to be explained with the principle of the Type II reversal in mind. This type of reversal usually takes place as a result of rotating a viewer or an object. In this case, however, both rotations are unnecessary to see the pencil's mirror image because the pencil is reflected in the mirror as it is. Although, strictly speaking, what is seen directly and what is seen in the mirror are not identical, this difference usually goes unnoticed because a pencil thus placed is virtually symmetrical with respect to its front and back. Since neither the viewer nor the pencil is rotated, no left-right reversal takes place.

The same account can be applied to a character written on a transparent sheet or one that is cut out of a sheet of paper. Both of them look normal (i.e., not reversed) in a mirror because they do not have to be rotated, or we do not have to turn around to see them in the mirror.

The differences between the locomotion hypothesis and the multiprocess hypothesis were discussed in the Type I Reversal and Type II Reversal sections. One of the remaining criticisms of the locomotion hypothesis is the one made by Block (1974): Pears (1952) failed to ex- 
plain why a viewer's body image has to walk rotationally, even though walking straight ahead is also an ordinary type of locomotion. In the account of the Type I reversal by the multiprocess hypothesis, the rotation of a representational frame has a convincing reason, which has already been stated. If it moved straight ahead, it could not be aligned with the mirror image's viewpoint.

Navon's (1987) criticisms of the locomotion hypothesis have already been discussed in The Case of Lying Down and The Case of a Mirror on a Ceiling or a Floor sections. The locomotion hypothesis shares with the symmetry hypothesis the problem that no reasonable basis has been provided for superimposing a viewer's body image onto the mirror image. This problem does not exist in the multiprocess hypothesis because the latter does not assume the superimposition of the body image.

The relation between the symmetry hypothesis and the multiprocess hypothesis (or the social learning hypothesis) was discussed in the Type I Reversal and Social Learning Hypothesis sections. In addition to the problem of superimposition, the symmetry hypothesis has difficulty in explaining the left-right mirror reversal of an alphanumeric character that is approximately symmetrical with respect to a horizontal axis but obviously asymmetric with respect to a vertical axis (e.g., C). The multiprocess hypothesis has no such difficulty because it does not resort to symmetry in explaining the mirror reversal of characters, which is classified into the Type II reversal in this hypothesis.

Neither the symmetry hypothesis nor the conventional description hypothesis can explain an up-down mirror reversal because both of them rely on the bilateral symmetry of a human body as a fundamental principle of explanation. The up-down reversal poses no problem for the multiprocess hypothesis because the latter does not rely solely on the bilateral symmetry; the up-down reversal is explained by a mirror's optical transformation (see the Type III-II Reversal, The Case of a Mirror on a Ceiling or a Floor, and The Case of Two Mirrors sections) or by a viewer's rotation about a horizontal axis (see the Type II Reversal section). Although some other phenomena (e.g., an up-down reversal seen in a concave mirror like a parabolic antenna) are left unexplained in this paper due to space limitations, it will not be difficult for the reader to find their proper explanations if the optical characteristics of the concerned mirrors (see Thomas, 1980) are combined with the appropriate principles of mirror reversal formulated in the multiprocess hypothesis.

The social learning hypothesis resembles the conventional description hypothesis in that it, too, refers to "convention." However, the meaning of this term is entirely different in these two hypotheses. In the conventional description hypothesis, it is a convention to call a mirror image "a left-right reversal." In the social learning hypothesis, it is a convention to employ an orientational framework that is aligned to a person's body in identifying its symmetric body parts with directional terms.
The conventional description hypothesis involves a leap in logic with regard to the reason why the bilateral symmetry of a human body leads to the verbal convention of "a left-right reversal." This criticism does not apply to the multiprocess hypothesis since it does not assume such a verbal convention.

Block (1974) criticized Gardner (1964) and Bennett (1970) in that they failed to explain why the left-right reversal is conventional and true in the case of a side view but conventional and false in the case of a frontal view. Aside from whether the terms true and false are adequate or not, the multiprocess hypothesis has a reasonable basis for discriminating between the side and frontal views: The former is explained as a Type III-I reversal in terms of a mirror's optical transformation, whereas the latter is explained as a Type I reversal in terms of the rotational transformation of a representational frame. As stated previously, the left-right reversal due to the optical transformation may well be called a "true" reversal.

The conventional description hypothesis cannot explain why the assumed verbal convention is not applied to real bodies. In the case of the multiprocess hypothesis, a mirror's optical transformation always plays an indispensable part in explaining any type of mirror reversal. Therefore, no reversal is predicted for real bodies.

The frontal encounter schema hypothesis has two major difficulties - the mirror reversal of alphanumeric characters, and that of a viewer's body in a mirror on a ceiling or a floor. Both reversals have already been given reasonable accounts by the multiprocess hypothesis.

In the Type II Reversal section, it was shown that none of the criticisms raised against the rotation hypothesis applies to the multiprocess hypothesis.

Thus, it is now clear that the multiprocess hypothesis has succeeded in avoiding all the difficulties associated with the preceding hypotheses. The multiprocess hypothesis combined with the social learning hypothesis can be considered to be valid on the following grounds: First, it has clarified the underlying principles of all the mirror reversals while properly combining the optical characteristics of mirrors with the transformations of orientational frameworks. Second, this hypothesis is consistent with what is commonly known about form recognition, mental imagery, and cognitive maps. Third, this hypothesis has not made questionable assumptions (e.g., a plane mirror selectively reverses left and right without reversing up and down). Finally, and above all, this hypothesis has succeeded in providing reasonable accounts for all the phenomena related to the mirror reversal within a consistent theoretical framework.

\section{REFERENCES}

BENNETT, J. (1970). The difference between right and left. American Philosophical Quarterly, 7, 175-191.

Biederman, I. (1987). Recognition-by-components: A theory of human image understanding. Psychological Review, 94, 115-147 
BLock, N. J. (1974). Why do mirrors reverse right/left but not up/ down? Journal of Philosophy, 71, 259-277.

Corballis, M. C., \& Beale, I. L. (1976). The psychology of left and right. Hillsdale, $\mathrm{NJ}$ : Erlbaum.

Gallistel, C. R. (1994). Space and time. In N. J. Mackintosh (Ed.), Animal learning and cognition (pp. 221-253). San Diego: Academic Press.

GARDNER, M. (1964). The ambidextrous universe. New York: Basic Books.

GARDNER, M. (1990). The ambidextrous universe (3rd ed.). New York: Charles Scribner's

Golledge, R. G. (1986). Environmental cognition. In D. Stokols \& I. Altman (Eds.), Handbook of environmental psychology (pp. 131174). New York: Wiley.

GREGORY, R. L. (1987). Mirror reversal. In R. L. Gregory (Ed.), The $O x$ ford companion to the mind (pp. 491-493). Oxford: Oxford University Press.

HaIG, N. D. (1993). Reflections on inversion and reversion. Perception, 22, 863-868.

Hinton, G. E., \& Parsons, L. M. (1981). Frames of reference and mental imagery. In A. Baddeley \& J. Long (Eds.), Attention and performance $I X$ (pp. 261-277). Hillsdale, NJ: Erlbaum.

ItTelson, W. H., MOWAFY, L., \& MAGID, D. (1991). The perception of mirror-reflected objects. Perception, 20, 567-584.

Kozlowski, L. T., \& Bryant, K. J. (1977). Sense of direction, spatial orientation, and cognitive maps. Journal of Experimental Psychol ogy: Human Perception \& Performance, 3, 590-598.

MARR, D. (1982). Vision. San Francisco: W. H. Freeman.

MARR, D., \& Nishihara, H. K. (1978). Representation and recognition of the spatial organization of three-dimensional shapes. Proceedings of the Royal Society of London: Series B, 200, 269-294.

MAYo, B. (1958). The incongruity of counterparts. Philosophy of Science, 25, 109-115.

MorRIs, R. C. (1993), Mirror image reversal: Is what we see what we present? Perception, 22, 869-876.

NAVON, D. (1987). Why do we blame the mirror for reversing left and right? Cognition, 27, 275-283.

PEARS, D. (1952). The incongruity of counterparts. Mind, 61, 78-81

PiageT, J., \& INHELDER, B. (1956). The child's conception of space (F. J.
Langton \& J. L. Lunzer, Trans.). London: Routledge \& Kegan Paul. (Original work published 1948)

Pylyshyn, Z. W. (1973). What the mind's eye tells the mind's brain: A critique of mental imagery. Psychological Bulletin, 80, 1-24.

Pylyshyn, Z. W. (1984). Computation and cognition: Toward a foundation for cognitive science. Cambridge, MA: MIT Press.

Rock, I. (1973). Orientation and form. New York: Academic Press.

Rock, I., SCHreiber, C., \& Ro, T. (1994). The dependence of twodimensional shape perception on orientation. Perception, 23, 14091426.

SHEPARD, R. N., \& COOPER, L. A. (1982). Mental images and their transformations. Cambridge, MA: MIT Press.

SHEPARD, R. N., \& HURwitz, S. (1984). Upward direction, mental rotation, and discrimination of left and right turns in maps. Cognition, $18,161-193$

SHEPaRd, R. N., \& METzleR, J. (1971). Mental rotation of threedimensional objects. Science, 171, 701-703.

TAKaNo, Y. (1989). Perception of rotated forms: A theory of information types. Cognitive Psychology, 21, 1-59.

Thomas, D. E. (1980). Mirror images. American Scientist, 243, 158172.

\section{NOTES}

I. In the first position, the viewer may rotate the representational frame to assume the real object person's viewpoint. Then, the watch is judged to be on the left. After turning around, the viewer may rotate the representational frame again to assume the mirror image's viewpoint. Then, the watch is judged to be on the right. The viewer will recognize a left-right reversal between these two judgments. In this case, the reversal results from the combination of two Type I reversals and one Type II reversal.

2. Note that the "front" of the character refers to its rear side in the present explanation. This naming was adopted because the rear for the character corresponds to the front for the viewer in the initial position.

(Manuscript received February 6, 1997; revision accepted for publication August 13, 1997.) 